\title{
EPIDEMIOLOGIA DA ATIVIDADE FÍSICA E A APROXIMAÇÃO NECESSÁRIA COM AS PESQUISAS QUALITATIVAS
}

\author{
DR. PEDRO CURI HALLAL \\ Pós-doutor do Programa de Pós-graduação em Epidemiologia e do Programa de \\ Pós-graduação em Educação Física \\ Grupo de Estudos em Epidemiologia da Atividade Física (GEEAF) \\ Universidade Federal de Pelotas (Pelotas - Brasil) \\ e-mail: prchallal@gmail.com
}

MS. ALAN GOULARTE KNUTH

Doutorando do Programa de Pós-graduação em Epidemiologia

Universidade Federal de Pelotas - Grupo de Estudos em Epidemiologia

da atividade física (GEEAF) (Pelotas - Brasil)

e-mail: alan_knuth@yahoo.com.br

\begin{abstract}
RESUMO
Os objetivos do presente ensaio foram: a) discutir os avanços da área de epidemiologia da atividade física no Brasil; b) destacar a necessidade de pesquisas qualitativas na área; c) apontar alguns caminhos de pesquisa que integram as diferentes metodologias. Temse observado um crescimento do campo de epidemiologia da atividade física no Brasil, especialmente a partir do ano 2000, que coincide com a maior inserção de profissionais de Educação Física nos programas de pós-graduação em saúde coletiva e epidemiologia. No entanto, esse crescimento se dá basicamente por meio de pesquisas de natureza quantitativa, visto que a maioria dos estudos qualitativos brasileiros na área têm se limitado a comentar os métodos utilizados nas pesquisas quantitativas e suas limitações.
\end{abstract}

PALAVRAS-CHAVE: Atividade motora; pesquisa qualitativa; epidemiologia; pesquisa quantitativa. 


\section{INTRODUÇÃO}

Brasil vem passando por processos de transição demográfica, nutricional e epidemiológica acelerados nos últimos anos. A transição demográfica tem como consequência o envelhecimento populacional, visto que as taxas de natalidade estão diminuindo e hoje se conhece maiores alternativas para reduzir ou estabilizar as principais causas de mortalidade infantil, além de um maior controle sobre as doenças infecto-contagiosas. Com as pessoas vivendo por mais tempo e um decréscimo no número de nascimentos, a população idosa está crescendo em ritmo acelerado no Brasil. A transição nutricional, que nos países de renda alta significou basicamente uma mudança da desnutrição para a obesidade como o principal problema nutricional, ocorre de forma complexa no Brasil, onde ambas as condições coexistem. Além disso, perfis nutricionais amplamente distintos são encontrados em diferentes regiões do Brasil. Por fim, a transição epidemiológica mostra que desde a metade do século passado houve uma diminuição proporcional marcante das doenças infecciosas e crescimento proporcional acelerado das doenças e agravos não-transmissíveis, com destaque (negativo) para as doenças cardiovasculares, os diferentes tipos de câncer e as causas externas, como acidentes e violências (MONTEIRO, 2000).

O conjunto dessas transições, que vem modificando os perfis de morbimortalidade, acaba colocando a atividade física na lista de prioridades da agenda de saúde pública nacional e internacional, visto que a atividade física está relacionada de forma causal com a ocorrência de diversas doenças e agravos não-transmissíveis (HASKELL, 2007). Com isso, a área de epidemiologia da atividade física cresceu rapidamente no cenário cientíico internacional. Como exemplo, pode-se citar que enquanto na década de 50, em torno de cinco artigos contendo a palavra "atividade física" no título ou resumo eram publicados na base de dados Pubmed por mês, esse número chegou a 2300 na década atual. Esse aumento não se deve apenas ao crescimento global da base de dados, visto que ocorre tanto em termos absolutos quanto em termos proporcionais.

Sabe-se, porém, que o acúmulo de conhecimento científico acerca dos benefícios da atividade física não é suficiente, de forma isolada, para gerar aumento nos níveis populacionais de atividade física (BORGES et al., 2009). A promoção da atividade física é complexa, e os novos conhecimentos produzidos sobre os seus benefícios são, até hoje, normalmente inócuos para gerar mudanças comportamentais contundentes no âmbito populacional. Parte dessa dificuldade reside na multicausalidade de tal comportamento, o qual está intimamente ligado a aspectos socioeconômicos, ambientais e culturais, além dos pessoais (TROST et al., 2002). Com base nessa complexidade, diversas instituições e pesquisadores brasileiros têm despendido esforços para a promoção da atividade física no país. 
Como o caminho científico de produção de conhecimento na área de atividade física relacionada com a saúde acabou se hospedando em centros de epidemiologia e saúde coletiva, as pesquisas desse campo acabam tendo um componente quantitativo bastante robusto. Por outro lado, a aproximação da pesquisa quantitativa com outras abordagens metodológicas parece ser um caminho ainda pouco explorado, porém promissor, principalmente se vincularmos essa produção ao campo da Educação Física e suas sub-áreas distintas, as quais claramente precisam estar mais conectadas.

Os objetivos do presente ensaio teórico foram: a) discutir os avanços atingidos na área de epidemiologia da atividade física no Brasil nas últimas décadas; b) valorizar a necessidade de pesquisas "qualitativas" na área, utilizando alguns exemplos nacionais e internacionais; c) apontar alguns caminhos de pesquisa que integrassem as diferentes metodologias. Deve-se deixar claro que a visão de pesquisa "qualitativa" versus. "quantitativa" como divisão hegemônica dos tipos de estudo é limitada, e tal segregação somente é utilizada nesse ensaio para facilitar a leitura. Por isso, os termos são utilizados entre aspas ao longo do texto.

\section{EVOLUÇÃO DA ÁREA DE EPIDEMIOLOGIA DA ATIVIDADE FÍSICA NO BRASIL}

A inserção de profissionais da Educação Física nos programas de pós-graduação em Saúde Coletiva e Epidemiologia ocorreu, de forma marcante, apenas a partir do ano 2000. Antes disso, tal colocação era rara e alguns programas sequer aceitavam a inscrição de profissionais com formação em Educação Física.

Um estudo de revisão documentou a evolução temporal da epidemiologia da atividade física no Brasil, até 2005 (HALLAL et al., 2007). Os autores buscaram identificar investigações epidemiológicas brasileiras, com amostras representativas de populações definidas e com pelo menos 500 indivíduos em estudo. Além de buscas eletrônicas em bases indexadas, os autores do referido estudo realizaram contatos com pesquisadores para mapear a distribuição das pesquisas nessa área no país. Foram localizados 42 trabalhos, com maior concentração de publicações a partir de 2000. Os pesquisadores também notaram uma disparidade regional desse conhecimento, pois até 2005 não encontraram estudos com dados exclusivos do Norte e Centro-Oeste do Brasil. Foram apontadas limitações do método epidemiológico de investigar a atividade física, como: (a) a repetição de estudos do tipo transversal a despeito de outros delineamentos; (b) a medição da atividade física por meio de questionários diferentes e, muitas vezes, não validados; (c) as múltiplas definições dos desfechos relacionados à prática de atividade física, e como consequência, a limitada comparabilidade entre diferentes estudos. 
Há que se notar que todo estudo epidemiológico de condução coerente, apesar da fidelidade aos métodos propostos, apresenta limitações. Faz parte do escopo dos bons trabalhos na área o apontamento de suas fragilidades. $O$ método epidemiológico tem uma responsabilidade enorme aplicado à temática da atividade física, mas não é capaz de responder, por si só, a todos os questionamentos da área.

Uma das facetas de aperfeiçoamento do método epidemiológico são os estudos comparativos. Apontamos nesse momento dois estudos comparativos, com objetivos distintos, mas que muito bem ilustram essa discussão. $O$ primeiro, conduzido em Pelotas, RS (KNUTH et al, 20 I 0) preenche os aspectos metodológicos deficitários verificados em estudos pontuais, de metodologias múltiplas. Os pontos fortes metodológicos do estudo passam pelo uso do mesmo instrumento de pesquisa, mesma delimitação do desfecho, mesma faixa-etária, mesmo local e domínios da atividade física em comparação com pesquisa realizada cinco anos antes.

Outra abordagem que merece destaque é o novato sistema brasileiro "Vigilância de fatores de risco e proteção para doenças crônicas por inquérito telefônico" - VIGITEL (BRASIL, 2009). Muito além do rigor acadêmico, o VIGITEL consolida a atividade física no plano institucional de saúde, em nível federal, num sistema de monitoramento que é novo no país. A aproximação desse sistema com a proposição de políticas públicas em saúde e outras áreas parceiras é hoje realidade e a atividade física está entre os aspectos a serem relevados.

A interlocução da atividade física com o Ministério da Saúde vai além. Desde a Política Nacional de Promoção da Saúde (BRASIL, 2006), editais e portarias anuais estimularam municípios e estados a promoverem estratégias populacionais em atividade física e outros temas, com abordagem integrada, intersetorial e de participação social. Nessas condições, foi formada uma Rede Nacional de Atividade Física (MALTA et al., 2008), que além de estar atrelada aos serviços de saúde locais, dialogando com o Ministério da Saúde, recebe apoio e parceria do Centro de Controle e Prevenção de Doenças Norte Americano, que trabalha em várias frentes com o Ministério da Saúde no Brasil e universidades parceiras.

Ponto alto dessa inserção brasileira na pesquisa e saúde institucional pôde ser observado no recente $3^{\circ}$ Congresso Internacional de Atividade Física e Saúde Pública, realizado em Toronto no Canadá, em 20 I0. Além de conduzirem falas e participações de destaque no evento, inclusive com uma mesa redonda exclusiva sobre a experiência brasileira na área, foi registrada a participação presencial de 29 pesquisadores brasileiros no congresso, o que coloca nosso país na posição de expoente na pesquisa epidemiológica em atividade física no contexto internacional. 


\section{CONTRIBUIÇÕES ATUAIS DA PESQUISA QUALITATIVA PARA A EPIDEMIOLOGIA DA ATIVIDADE FÍSICA NO BRASIL}

A epidemiologia é uma das ciências básicas da saúde pública. A aproximação da atividade física com esse campo fortalece a área, mas claramente o método epidemiológico não responde a todas as questões de pesquisa na temática. A epidemiologia é robusta para avaliar a frequência, distribuição e determinantes dos processos saúde-doença nas populações. No entanto, quando há a intenção de compreender o "porquê" de um determinado fenômeno, seus métodos, por vezes, chegam a um limiar de saturação, onde outras abordagens associadas podem complementar o olhar epidemiológico.

Uma das questões atuais em saúde pública e de relação imediata com a prática de atividade física é o uso de anabolizantes em frequentadores de academias de ginástica. Essa conduta que tem como um dos propulsores os padrões estéticos corporais atuais poderia ser "mapeada" por meio de estudos epidemiológicos. No entanto, com a tradicional abordagem de questionários, rotineiramente diretos e com questões fechadas e rápidas, já com o propósito de análise, esse método não seria ideal para a compreensão aprofundada do tema em estudo. Assim, uma investigação em Salvador, na Bahia (IRIART; CHAVES; ORLEANS, 2009), de cunho etnográfico, por meio de observação participante junto à frequentadores de academia merece destaque. $\bigcirc$ estudo dialogou com um tema em saúde pública, passível de receber uma abordagem epidemiológica, mas teve outro olhar singular e relevante para a epidemiologia da atividade física. As entrevistas aprofundadas com 43 usuários de anabolizantes oferecem inúmeras contribuições para a área.

A atuação epidemiológica no campo da atividade física é exaustivamente tachada de biologicista. Um dos campos macros em que a atividade física pode ser abordada e tratada como aspecto relacionado à saúde é o espaço escolar. Temos a clara convicção de que a abordagem escolar centrada na aptidão física é objeto inerente ao fracasso, ainda mais se apresentada com caráter doutrinador e descontextualizado ao contexto escolar de intervenção. A atividade física proposta na escola deve ser carregada de simbologias dos determinantes sociais, econômicos, políticos, culturais e ambientais. Por isso, o ensaio de Ferreira (200 I) é mais um exemplo de que o conhecimento biológico da atividade física e da epidemiologia não deve ser negado, mas complementado com a visão "qualitativa". O autor propõe abordagens para conduzir os temas atividade física e saúde em aulas escolares, relevando a sua importância biológica, porém ampliando o enfoque. Acreditamos que esse tipo de debate é de grande relevância, visto que, em nosso entendimento, as duas abordagens são complementares. 
Sem dúvida a maior compilação de ensaios buscando refletir sobre a atividade física, epidemiologia e saúde no Brasil está documentada em "A Saúde em Debate na Educação Física" (BAGRICHEVSKY; PALMA; ESTEVÃO, 2003; BAGRICHEVSKY et al., 2006; BAGRICHEVSKY; ESTEVÃO; PALMA, 2007). Vários debates e esforçOs com possibilidades teórico-metodológicas de abordar temas como o corpo e a saúde são fixados nas publicações. É possível encontrar na apresentação da segunda edição o seguinte aspecto:

\begin{abstract}
...é necessário reconhecer, de pronto, que as aspirações (pretensamente críticas) do presente livro - resultado do envolvimento generoso do seu coletivo de participantes - talvez, sejam muito maiores, do que nossa capacidade de responder às próprias questões que produzimos e compartilhamos com o leitor. Esta espécie de justificativa introdutória cumpre a função de alertar aos incautos que os ensaios aqui apresentados, provavelmente, não servem a anseios 'prescritivos' e 'solucionadores' de problemas epistemológicos e praxiológicos em saúde.
\end{abstract}

Ao mesmo tempo em que os autores assumem a limitação de seus ensaios, seria importante que fosse entendido que a área "quantitativa" também tem limitações. No entanto, deve-se deixar claro que a maioria das investigações epidemiológicas em atividade física não procura ter caráter prescritivo. $\bigcirc$ conhecimento produzido pelo campo "quantitativo" não é definitivo, pronto ou superior a outros modelos de investigação. $\bigcirc$ que a área de atividade física precisa é de fortalecimento. Em nossa visão, ainda pouco se produziu no campo das abordagens "qualitativas".

O debate entre as duas áreas precisa transpor o eixo de disputa discursiva. Uma ilustração para esse tom é o conceito de "demonização do sedentarismo" colocado recentemente (BAGRICHEVSKY; ESTEVÃO; VASCONCELLOS-SILVA, 2007, p. 862). Estamos de acordo com os autores quando perguntam: “...como fazê-lo sem estigmatizar os sujeitos classificados como sedentários?", referindose a um possível combate do sedentarismo, que nós preferimos abordar como promoção da atividade física. De fato, algumas pesquisas epidemiológicas podem tratar a pauta sob essa perspectiva, mas muitos pesquisadores estão atentos para os problemas de culpabilização da vítima e querem avançar na abordagem do tema. A metodologia "qualitativa" poderia ser utilizada para auxiliar na definição da melhor forma de se relatar baixos níveis de atividade física, sem o uso de termos taxativos e traumatizantes, ao invés de apenas criticar os termos usados pelos pesquisadores "quantitativos". Assim, seguiríamos em debate, mas de forma propositiva (errando, eventualmente), procurando avançar.

Assim, deve-se sempre evitar o "caráter moralizador" recentemente atribuído às pesquisas epidemiológicas na área da atividade física (PALMA; VILAÇA, 20 I 0). 
○ "fardo moral" que pode ser resultante da "epidemia do sedentarismo" deve ser objeto frequente de reflexão. As pesquisas epidemiológicas não são irrefutáveis em suas classificações, porém isso não afasta a possibilidade de inquéritos em saúde medirem ou se aproximarem de uma medida de qualidade dos níveis de atividade física nas populações. As definições usadas em estudos epidemiológicos para inativos, sedentários, insuficientemente ativos são muito mais da ordem de análise, mas com certeza podem ser repensadas. Concordamos que elas podem, se mal empregadas ou interpretadas, parecerem taxativas ou traumatizantes.

Como anteriormente mencionado o método epidemiológico é passível de aperfeiçoamento. Deve-se deixar claro que a área de atividade física está aberta para contribuições de pesquisadores com diferentes abordagens, e como todo tema em crescimento, desperta interesses e dualidades em seu entendimento.

\section{EXEMPLOS DOS AUTORES DE PESQUISAS COMBINANDO METODOLOGIAS QUANTITATIVAS E QUALITATIVAS}

Na área de avaliação de intervenções em atividade física, destaca-se o estudo combinando metodologias "quantitativas" e "qualitativas" sobre programas de promoção da atividade física nas cidades de Recife (PE) e Curitiba (PR). A combinação de métodos permitiu que os programas fossem avaliados integralmente, de forma que fossem ouvidos usuários, não-usuários, gestores e profissionais envolvidos com as intervenções (HALLAL et al., 20 I 0; HALLAL et al., 2009; HALLAL et al., 2009b). Tensões entre forças gestoras dos programas e equipe de avaliação, impossíveis de serem captadas no estudo "quantitativo", foram claramente identificadas no estudo "qualitativo", especialmente em Recife (PE).

$\mathrm{Na}$ cidade de Pelotas (RS), no estudo de coorte que acompanha os nascidos em 1993, estudos com delineamento epidemiológico "quantitativo" e etnográfico foram conduzidos com jovens de I I anos de idade. Determinantes da prática de atividade física nessa faixa etária detectados na pesquisa "quantitativa" foram aprofundados na pesquisa "qualitativa" (GONÇALVES et al., 2007). O estudo etnográfico detectou que os meninos têm maior apoio social e familiar para a prática de atividade física nessa idade, o que pode explicar os maiores níveis de atividade física observados em meninos quando comparados às meninas nos estudos "quantitativos".

Recentemente, foi publicado um artigo sobre as lições aprendidas após 10 anos de uso do Questionário Internacional de Atividade Física (IPAQ) no Brasil e na Colômbia (HALLAL et al. , 20 l 0b). Destaca-se aqui o trabalho realizado por pesquisadores colombianos, os quais, por meio de metodologias de pesquisa qualitativa, especialmente grupos focais, avaliaram o grau de compreensão do instrumento pelos 
habitantes daquele país. Foram propostas mudanças no instrumento com base na avaliação qualitativa, de forma que o instrumento atualmente usado no país está mais adaptado à realidade local.

Limitamo-nos a apresentar alguns estudos nos quais tivemos contribuição direta para evitar tornar essa seção demasiadamente longa. $\bigcirc$ objetivo é apenas ilustrar os tipos de estudos que podem ser feitos quando as duas metodologias trabalham de forma integrada.

\section{ÁREAS PRIORITÁRIAS PARA PESQUISAS QUALITATIVAS EM EPIDEMIOLOGIA DA ATIVIDADE FÍSICA}

Uma das principais limitações da área de epidemiologia da atividade física se refere à conceituação, visto que existe uma abundância de termos utilizados para classificar os indivíduos de determinado estudo em termos de sua prática de atividade física, como já mencionado neste ensaio. $\bigcirc$ uso de rótulos normativos na área de saúde pública é comum (indivíduo obeso, hipertenso), mas claramente existe a necessidade de mudança desse paradigma. Pesquisas "qualitativas" podem auxiliar na definição dos termos utilizados na área de epidemiologia da atividade física, e na proposição de classificações mais "amenas".

Embora tenha havido um rápido crescimento de métodos diretos para mensuração de atividade física em pesquisas epidemiológicas nos últimos anos (acelerômetros, pedômetros), a grande maioria dos estudos, especialmente nos países de renda média ou baixa, ainda utiliza questionários para avaliar a prática de atividade física. Tais instrumentos geralmente foram desenvolvidos em países de renda alta, e sua aplicabilidade para a realidade brasileira deve ser testada. Conforme mencionado anteriormente, com o exemplo colombiano, pesquisadores com enfoque "qualitativo" poderiam auxiliar na construção de instrumentos de pesquisa adaptados à realidade brasileira.

No que se refere aos fatores associados à prática de atividade física, alguns autores têm conduzido estudos na área de barreiras percebidas para a prática de atividade física (REICHERT et al., 2007). É evidente que o uso de metodologias de pesquisa "qualitativa" pode nos fornecer informações valiosas para a compreensão dos principais fatores que dificultam a adesão ou manutenção da prática de atividade física. Tão importante ou mais do que conhecer as barreiras para a prática de atividade física, seria interessante que estudos "qualitativos" fossem conduzidos com pessoas ativas, para que a área conhecesse também o que motiva tais pessoas a incorporarem a atividade física no seu dia-a-dia. Ao que nos parece, a condução de abordagens com grupos focais poderia ser explorada, e a análise dos indivíduos ativos, ou grupos específicos, como os idosos, podem engrandecer a área. 
Na área de intervenções em promoção da atividade física, estudos "qualitativos" também são urgentemente necessários. Com mais de 1000 municípios no país sendo financiados pelo Ministério da Saúde para o desenvolvimento de programas de promoção da atividade física, um campo imensurável de pesquisa está aberto. Compreender as razões que fazem com que algumas das intervenções consigam tornar as pessoas mais ativas, enquanto outras não conseguem, seria uma valiosa contribuição da pesquisa "qualitativa" para a área.

Por fim, não se deve ignorar o que vem sendo feito até agora. A constante leitura detalhada do que vem sendo produzido pelos pesquisadores "quantitativos" é necessária e bem vinda. Com esse texto, pretende-se deixar claro que essa função é recíproca, e os pesquisadores da área "quantitativa" também devem analisar atentamente o que vem sendo produzido por seus pares da área "qualitativa". A ideia desse ensaio é iniciar esse processo.

\section{CONSIDERAÇÕES FINAIS}

Nossa experiência de aproximação com a metodologia "qualitativa" é ainda recente e limitada. As dicas de atuação no campo da atividade física são oriundas de nossas impressões, resultantes de carências latentes percebidas com a pesquisa na área de epidemiologia da atividade física. A possibilidade de inclusão de diferentes formas de compreender os resultantes dos estudos de atividade física depende também da contundência da produção do conhecimento da área qualitativa. Acreditamos em uma produção compartilhada e propositiva, fazendo o campo de atividade física e saúde prosperar e ampliar sua forma de compreensão dos desfechos em saúde.

Recentemente muitos ensaios teóricos têm focado em críticas sobre os métodos epidemiológicos aplicados à atividade física, muitos dos quais foram discutidos neste ensaio. Imaginamos que essa abordagem possa amadurecer, e que, além disso, uma conduta propositiva também faça parte do escopo. Nenhum autor da epidemiologia poderá ignorar as limitações do método que usa e certamente saberá compreender abordagens alternativas ou caminhos distintos na compreensão dos fenômenos em saúde. O próprio estabelecimento de um chamamento ao debate à construção coletiva, incluindo novas formas de olhar os aspectos da atividade física já configura um possível avanço e uma inquietação em aceitar outros caminhos metodológicos que auxiliem na promoção da atividade física, considerando que a produção do conhecimento é um espaço intocável nesse desafio.

Por fim, a maioria das experiências e sugestões de abordagem "qualitativa" aqui mencionadas surgiram de pesquisas "quantitativas" anteriores. Seria ainda mais desafiador se os pesquisadores da área "qualitativa" pudessem pautar a epidemiologia sobre 
que áreas foram por eles identificadas como limitadas e passíveis de novos estudos. Essa troca nos parece enriquecedora e desde já dotada, de muita expectativa.

\section{Physical activity and the need of qualitative research}

ABSTRACT: The aim of the present essay were: a) to discuss the achievements of the epidemiology of physical activity field over the last decades in Brazil; b) highlight the need of qualitative studies in the field, by using some national and international examples; c) suggest some topics that could be explored in future studies using combined quantitative and qualitative methodologies. The field of physical activity epidemiology has grown substantially in Brazil over the last years, particularly after 2000, which coincides with the dates in which Physical Education teachers started to join postgraduate programs in Public Health and Epidemiology. However, these improvements were achieved based primarily on quantitative studies, given the fact that most qualitative studies in the field in Brazil have failed to go beyond commenting on the methods used in quantitative studies and its limitations. KEY WORDS: Motor activity; qualitative research; epidemiology; quantitative research.

\section{Actividad física y la necesidad de investigación cualitativa}

RESUMEN: Los objetivos de este ensayo son: a) discutir los alcances del campo de la epidemiologia en actividad física en Brasil durante las últimas décadas; b) destacar la necesidad de investigación cualitativa en esta área; se dan algunos ejemplos a nivel nacional e internacional; c) sugerir algunos tipos de investigaciones integrando las diferentes metodologías. El campo de la epidemiologia en actividad física en Brasil ha crecido significativamente durante los últimos años, pero particular a partir del 2000 que coincide con la fecha en la cual los profesores de educación física comenzaron a unirse a programas en salud pública y epidemiologia. Sin embargo, esta mejoría ha sido alcanzada principalmente gracias a estudios cuantitativos, mientras estudios cualitativos en el área se han limitado a comentar sobre los métodos usados en estudios cuantitativos y sus limitaciones.

PALABRAS CLAVES: Actividad motora; investigación cualitativa; epidemiologia; investigación cuantitativa.

\section{REFERÊNCIAS BIBLIOGRÁFICAS}

BAGRICHEVSKY, M.; PALMA, A.; ESTEVÃO, A. (Orgs.). A saúde em debate na educação física. Blumenau: Edibes, 2003.

BAGRICHEVSKY, M. et al. (Orgs.). A saúde em debate na educação física. Volume 2. Blumenau: Nova Letra, 2006.

BAGRICHEVSKY, M.; ESTEVÃO, A.; PALMA, A. (Orgs.). A saúde em debate na educação física. Volume 3. Ilhéus: Editora da UESC, 2007. 
BAGRICHEVSKY, M.; ESTEVÃO, A.; VASCONCELLOS-SILVA, P. R. Sedentarismo, nós e o mundo (im)possível no contexto da ciência. Revista de Saúde Pública, São Paulo, v. 4I , n. 5 , p. 862-64, out. 2007.

BORGES, T. et al. Conhecimento sobre fatores de risco para doenças crônicas: estudo de base populacional. Cadernos de Saúde Pública, Rio de Janeiro, v. 25, n. 7, p. I 5 I I-20, jul. 2009.

BRASIL. MINISTÉRIO DA SAÚDE. Vigilância de doenças crônicas por inquérito telefônico (Vigitel Brasil 2008). Estimativas sobre freqüência e distribuição sócio-demográfica de fatores de risco e proteção para doenças crônicas nas capitais dos 26 estados brasileiros e no Distrito Federal em 2008. Série G. Estatística e informação em saúde. Brasília: Ministério da Saúde, 2009.

BRASIL. MINISTÉRIO DA SAÚDE. Política Nacional de Promoção da Saúde. Secretaria de Vigilância em Saúde. Ministério da Saúde, Secretaria de Atenção à Saúde. - Brasília: 2006.

FERREIRA M. S. Aptidão física e saúde na educação física escolar: ampliando o enfoque. Revista Brasileira de Ciências do Esporte, Campinas, v. 22, n. 2, p. 4I-54, jan. 2001.

GONÇALVES, H. et al. Fatores socioculturais e nível de atividade física no início da adolescência. Revista Panamericana de Saúde Pública, Lawrence, v. 22, n. 4, p. 246-53, out. 2007.

HALLAL, P. C. et al. Evolução da pesquisa epidemiológica em atividade física no Brasil: revisão sistemática. Revista de Saúde Pública, São Paulo, v. 4I , n. 3, p. 453-60, jun. 2007.

HALLAL, P. et al. Avaliação do programa de promoção da atividade física Academia da Cidade de Recife, Pernambuco, Brasil. Cadernos de Saúde Pública, Rio de Janeiro, v. 26, n. I, p. 70-78, jan. 2010.

HALLAL, P. et al. Avaliação quali-quantitativa do programa Academia da Cidade, Recife (PE): concepções dos professores. Revista Brasileira de Atividade Física e Saúde, Londrina, v. I4, n. 2, p. 9-14, jun. 2009.

HALLAL, P. et al. Avaliação de programas comunitários de promoção da atividade física: o caso de Curitiba-PR. Revista Brasileira de Atividade Física e Saúde, Londrina, v. I4, n.2, p. I04-14, jun. 2009.

HALLAL, P. et al. Lessons learned after 10 years of IPAQ use in Brazil and Colombia. Journal of Physical Activity and Health, Champaign, v. 7, suplemento 2, p. 259-64, jul. 2010.

HASKELL, W. et al. Physical activity and public health: updated recommendation for adults from the American College of Sports Medicine and the American Heart Association. Medicine and Science in Sports and Exercise, Filadélfia, v. 39, n. 8, p. 1423-34, ago. 2007.

IRIART, J. A. B.; CHAVES, J. C.; ORLEANS, R. G. Culto ao corpo e uso de anabolizantes entre praticantes de musculação. Cadernos de Saúde Pública, Rio de Janeiro, v. 25, n. 4, p.773-782, abr.2009. 
KNUTH, A. G. et al. Changes in physical activity among Brazilian adults over a 5-year period. Journal of Epidemiology and Community Health, Londres, v. 64, p. 59I-595, ago. 2010.

MALTA D. C. et al. A promoção da atividade física no Sistema Único de Saúde. Revista Brasileira de Atividade Física e Saúde, Londrina, v. I3, n I, p. 24-27, mar. 2008.

MONTEIRO, C. A. Velhos e novos males da saúde no Brasil: a evolução do país e de suas doenças. 2a edição. São Paulo: Hucitec, 2000.

PALMA, A.; VILAÇA, M. M. O sedentarismo da epidemiologia. Revista Brasileira de Ciências do Esporte, Campinas, v. 31 , n. 2, p. 105-19, jan. 2010.

REICHERT, F. F. et al. The role of perceived personal barriers to engagement in leisuretime physical activity. American Journal of Public Health, Washington, v. 97, n. 3, p. 5I 5-9, mar. 2007.

TROST, S. et al. Correlates of adult's participation in physical activity: review and update. Medicine and Science in Sports and Exercise, Filadélfia, v. 34, n. 12, p. 1996-2001, dez. 2002.

Recebido: 21 mai. 2010 Aprovado: 17 dez. 2010

Endereço para correspondência:

Pedro Curi Hallal Rua Marechal Deodoro I 160, $3^{\circ}$ piso Bairro Centro, Pelotas - RS

CEP: $96020-220$ 